\title{
New Section on Crystallography Education
}

From our participation within the ICDD, also as instructors in workshops and courses and (dare we add) referees, it is clear that the scope of powder diffraction is expanding considerably. This has been largely (though not exclusively) driven by greater range of applications of Rietveld analysis and related full-pattern fitting techniques. Hugo Rietveld developed his method in the late 1960's to perform crystallographic analysis using neutron powder diffraction data, despite the unavoidable ambiguities that result from reflection overlap. Since that time, Rietveld analysis and related techniques have become widely used for X-ray diffraction and have expanded beyond the bounds of crystallographic analysis, and are used for materials characterization in areas such as residual stress, crystallite size and phase quantification. The technique has entered the mainstream within fields as disparate as condensed matter physics, pharmaceutical characterization, catalysis, protein crystallography, geosciences and materials engineering. The range of measurement strategies is staggering, with many new types of optics and detectors applied in laboratory-based instruments; even greater developments have occurred in synchrotron measurements, allowing routine access to resolution in the $\Delta Q / Q$ range of $10^{-4}$, and where pattern collection may be limited by the data transfer rates of detectors; neutron diffraction instrumentation has also matured and advanced instruments are available in many facilities.

Despite this growth in use and applicability, it seems that fewer colleges and universities are able to provide in-depth instruction in powder diffraction techniques and in

Note from the Editor-in-Chief:

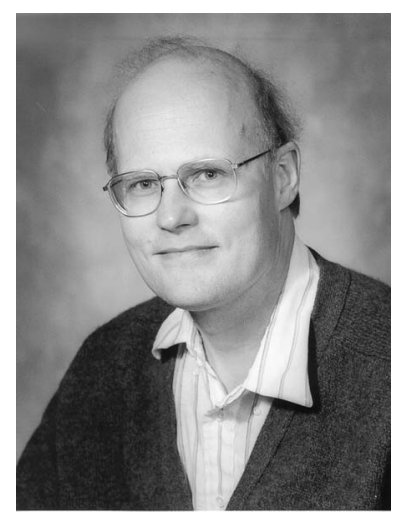

Dr. James A. (Jim) Kaduk has run the crystallographic service laboratory at Innovene USA (now part of INEOS; formerly BP and Amoco) in Naperville, IL for over 20 years. crystallographic analysis. Thus, many researchers and students must apply self-taught skills to function in these fields. One only needs to lurk within the Rietveld users' mailing list (Rietveld_1@ill.fr) to see people all across the world asking the same questions and tripping over the same bumps in the road. This is a problem for experts as well as beginners; advanced aspects of Rietveld analysis have been documented only minimally.

We are rash enough to think that we can help the situation by providing tutorial and instructional articles in a new Journal section specific to educational materials related to powder diffraction crystallography. As editors for this section, we wish to foster creation of educational materials covering topics not found in books or manuals. Furthermore, a new range of computer-assisted technologies is advancing the methods available for instruction, and we hope to see articles that marry the traditional (journal pages) and the new (web pages, streaming video and more).

While we plan to write some of these articles ourselves, we encourage others to do so as well. Unsolicited articles will be considered for publication, but we encourage potential authors to contact us in advance to discuss their ideas in advance of manuscript completion. All articles will be reviewed prior to publication.

\section{Brian Toby \\ Argonne National Laboratory \\ Jim Kaduk \\ Innovene USA}

He characterizes a wide variety of materials, including catalysts, rusts and cruds, polymers, coordination complexes, and small organic molecules. He does virtually all of his data processing by the Rietveld method, solving the crystal structures when necessary. He is an active user of synchrotons and neutron sources to solve practical problems, and applies computational chemical techniques to complement his crystallographic studies. He is currently Chairman of the Board of Directors of ICDD, Chair of the U. S. National Committee for Crystallography, and a member of the Denver X-ray Conference Organizing Committee. He is a Co-Editor of Acta Crystallographica B: Structural Science. He is a member of the faculties of the ICDD Clinic on Advanced Methods in X-ray Powder Diffraction, the ACA summer schools on small-molecule and macromolecule crystallography, and teaches at workshops and short courses in powder diffraction worldwide. 


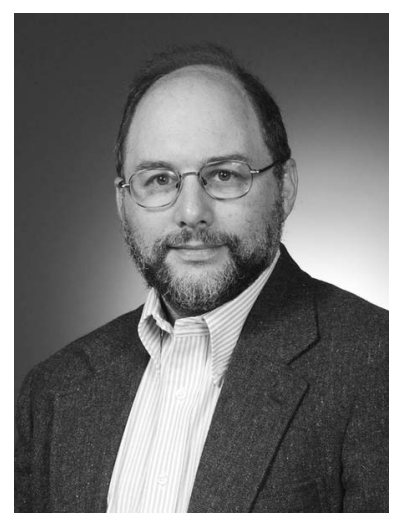

Dr. Brian Toby is a group leader at the Advanced Photon Source of Argonne National Laboratory, where he is heading development of a very high-resolution and high-throughput robot-enabled powder diffractometer, as well as programs in high energy and surface diffraction. Since receiving his Ph.D. in 1986, he has worked with powder diffraction while employed in one academic lab, two industrial labs and two government labs, primarily working with synchrotron and neutron instrumentation. He is a Fellow of the International Centre for Diffraction Data, where he has chaired several subcommittees and a task force; he is active within the American Crystallographic Association and has been a member of two International Union of Crystallography commissions. He currently serves on the US National Committee for Crystallography. His research interests include structure refinement and determination techniques, informatics for powder diffraction and crystallographic studies of functional metal oxides and zeolitic materials. He has coauthored nearly 100 refereed papers. In 2005, he received a Bronze Medal from the U.S. Department of Commerce for developing several popular crystallographic software packages, CMPR, CIFTOOLS (pdCIFplot) and EXPGUI. He is also the principal author of the international standard for communication of powder diffraction data and Rietveld results, pdCIF.

Ting C. Huang Editor-in-Chief 